\title{
Die Frau fürs Pädagogische
}

BEATE KLEMME Ihr beruflicher Werdegang begann ursprünglich in der Pädagogik.

Über die Sportwissenschaften kam Beate Klemme dann zur Physiotherapie.

Heute verbindet die Professorin ihre Kompetenzen und bildet an der Fachhochschule

Bielefeld Physio- und Ergotherapeuten zu qualifizierten Fachlehrern aus. Sie ist

davon überzeugt, dass Lehrende spezifische Kompetenzen benötigen.

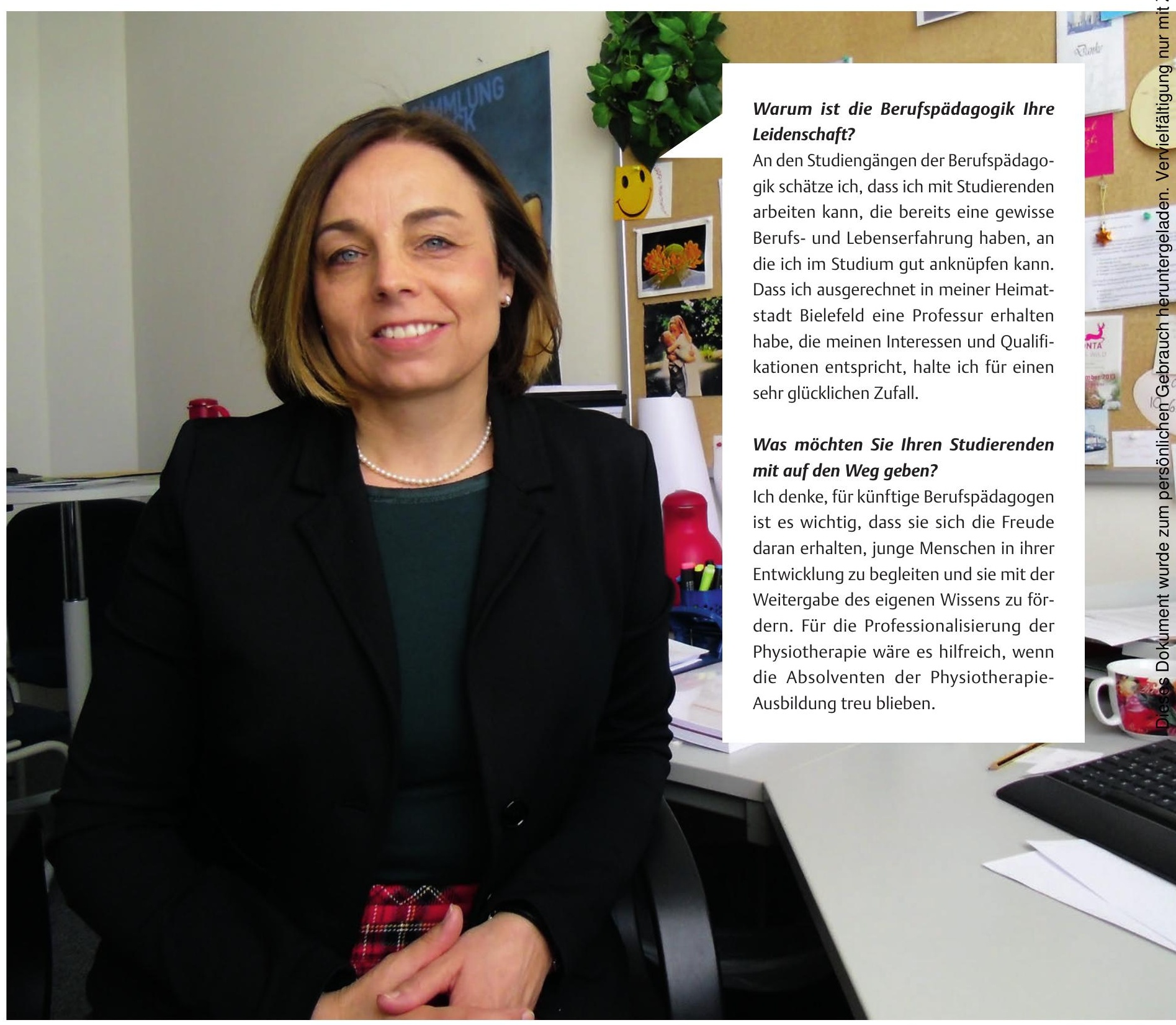




\section{Lehrer spezifisch qualifizieren}

\section{Das Tätigkeitsfeld}

In Deutschland gibt es für die Ausbildung von Lehrkräften in Therapieberufen keine bundesweit einheitliche Regelung. Während in den neuen Bundesländern der Abschluss eines pädagogischen Universitätsstudiums erforderlich ist, genügt in den alten Ländern die Berufszulassung als Voraussetzung für die Lehrtätigkeit an einer Berufsfachschule. Auch die Ausbildung der Anleiter in der praktischen Ausbildung ist nicht geregelt. Doch das Bewusstsein, dass eine hochschulische Ausbildung für die Lehre sinnvoll ist, ist in den Therapieberufen inzwischen gewachsen. Seitens der Politik fehlt in den alten Bundesländern jedoch ein klares Bekenntnis zur akademischen Lehrerbildung. Das liegt unter anderem daran, dass die Berufsfachschulen in den Bundesländern unterschiedlichen Ministerien unterstellt sind und dadurch eine einheitliche Vorgehensweise erschwert wird. Seit vielen Jahren engagieren sich vereinzelt Berufsangehörige für eine bessere Qualifizierung der Lehrenden. So auch Beate Klemme. Als sie ihre Professur an der Fachhochschule Bielefeld antrat, erhielt sie den Auftrag, sich mit der Fachdidaktik der Physiotherapie auseinanderzusetzen. Keine leichte Aufgabe, denn für die Konzeption entsprechender Studienmodule gab es im deutschsprachigen Raum keine Literatur. Die Herausforderung bestand darin, eine Balance zwischen Konzepten aus den Nachbardisziplinen, wie der Pflege, und eigenen Strukturen zu finden. Interessante Impulse, insbesondere zum Clinical Reasoning, holte sie sich zudem aus den Ländern Australien und Kanada. So schaffte sie in Bielefeld ein einzigartiges berufspädagogisches Bildungsangebot. Das Bachelorstudium ist auf sechs Semester angelegt (www.fh-bielefeld.de/studienangebot). In Modulen wie „Modelle der Therapiewissenschaften“ oder „Fachbezogene Forschung und Evidence Based Practice“ erweitern und vertiefen Physio- und Ergotherapeuten ihr Wissen in den Bereichen Therapie und Gesundheit. Zudem beschäftigen sie sich mit der Grundlage der Berufspädagogik, der Didaktik und der Psychologie beruflichen Lehrens und Lernens. Der anschließende Master setzt einen mit mindestens „gut“ bewerteten Bachelorabschluss in den Bereichen Pflege, Physio- oder Ergotherapie voraus und dauert vier Semester. In ihm vertiefen die Studierenden ihre pädagogischen, fachwissenschaftlichen und organisatorischen Kompetenzen und beschäftigen sich mit den Themen Schulentwicklung und -organisation und Bildungsforschung. Beate Klemme kann heute auf eine 13-jährige Erfahrung in der Lehrerbildung zurückblicken.

\section{Erkenntnisse}

Beate Klemme gewann aus ihrer bisherigen Arbeit die Erkenntnis, dass ...

> die Absolventen der berufspädagogischen Studiengänge sehr unterschiedlich in den Berufsfachschulen aufgenommen werden. Ihre Kompetenzen werden vom Lehrerkollegium teilweise geschätzt, teilweise aber auch kritisch betrachtet.

> die Absolventen in der Berufseinstiegsphase oft nicht als Anfänger wahrgenommen, sondern von Beginn an als Experten eingestuft und angesprochen werden. Ihnen fehlt eine Schonzeit, um sich in ihrem neuen Beruf auszuprobieren.

> es erforderlich ist, die Studierenden intensiv auf mögliche Schwierigkeiten der Rollenfindung in der Praxis vorzubereiten.

$>$ Absolventen, die sich bereits in einer Berufsfachschule etabliert haben, oft den Weg für die Einstellung weiterer Berufspädagogen ebnen.

\section{Fazit}

Zusammenfassend kann Beate Klemme festhalten, dass ...

> die Lehre und Ausbildung in den Therapieberufen spezifische Kompetenzen und damit eine umfassende hochschulische Qualifizierung erfordern. Deshalb plädiert sie für eigenständige berufspädagogische Studiengänge in diesem Feld.

> die Qualifizierung sich an sonst üblichen akademischen Lehrerausbildungen orientieren muss. Damit würden die Therapieberufe ihren Status als ewiger „Sonderfall“ in der berufspädagogischen Fachwelt überwinden. Eva Trompetter 